\title{
Morphologic and histologic findings in bioprosthetic valves explanted from the mitral position in children younger than 5 years of age
}

\author{
Laura Gellis, MD, ${ }^{\mathrm{a}}$ Christopher W. Baird, MD, ${ }^{\mathrm{b}}$ Sitaram Emani, MD, ${ }^{\mathrm{b}}$ Michele Borisuk, MS, MSN, \\ Kimberlee Gauvreau, SCD, ${ }^{\mathrm{a}}$ Robert F. Padera, Jr, MD, PhD, ${ }^{\mathrm{c}}$ and Stephen P. Sanders, $\mathrm{MD}^{\mathrm{a}}$
}

\section{ABSTRACT}

Background: Mitral valve replacement (MVR) in very young children is challenging. This study investigates the mechanisms for early bioprosthetic valve failure in very young patients through review of the macroscopic and microscopic findings in explanted bioprosthetic valves.

Methods: Patients who underwent MVR with a bioprosthetic valve at Boston Children's Hospital between January 2010 to April 2016 at $<5$ years of age were the subjects of this study. Valve failure was defined as prosthetic mitral valve explantation with mitral valve re-replacement.

Results: Bioprosthetic valves were used in 31 of 77 MVRs during the study period. Valve failure occurred in 10 patients $(32 \%)$. Freedom from valve failure was $80 \%$ at 1 year and was associated with older age at implantation. On gross and microscopic evaluation, valve failure (predominantly stenosis) was found to be due to pannus deposition and intrinsic leaflet calcification.

Conclusions: Successful long-term use of bioprosthetic valves in the mitral position in very young children continues to be a challenge. In addition to intrinsic calcification, excessive pannus deposition can lead to early bioprosthetic valve failure in this population. Early exuberant pannus growth appears due to thrombus deposition on the valves themselves and to the host's reaction to foreign material. (J Thorac Cardiovasc Surg 2018;155:746-52)

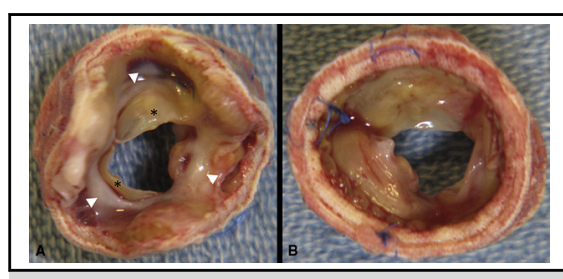

Porcine bioprosthesis, explanted after 13 months in situ, covered by thick pannus.

\section{Central Message}

Excessive pannus deposition can lead to early mitral bioprosthetic valve failure in young children. This appears to occur in response to thrombus deposition and the host's reaction to foreign material.

\section{Perspective}

In addition to the problem of intrinsic calcification, bioprosthetic valves in the mitral position pose the risk of early valve failure because of excessive pannus deposition in young children. The mechanisms of pannus formation (predominantly organization of small thrombi) suggest potential management strategies, such as a brief period of anticoagulation, that may prolong durability in young patients.

See Editorial Commentary page 753.
Mitral valve replacement (MVR) in very young patients is challenging. ${ }^{1-4}$ Valve sizes appropriate for small hearts are extremely limited and might not offer favorable hemodynamic characteristics. ${ }^{2,5,6}$ In addition, rapid somatic growth leads to patient-prosthesis

From the Departments of a Cardiology and b Cardiac Surgery, Boston Children's Hospital; and ${ }^{\mathrm{c}}$ Department of Pathology, Brigham and Women's Hospital, Boston, Mass.

Received for publication April 4, 2017; revisions received Sept 1, 2017; accepted for publication Sept 17, 2017; available ahead of print Oct 25, 2017.

Address for reprints: Stephen P. Sanders, MD, Department of Cardiology, Boston Children's Hospital, 300 Longwood Ave, Boston, MA 02115 (E-mail: Stephen. sanders@cardio.chboston.org).

$0022-5223 / \$ 36.00$

Copyright (c) 2017 by The American Association for Thoracic Surgery

https://doi.org/10.1016/j.jtcvs.2017.09.091 mismatch, further reducing the longevity of prosthetic valves in this population. ${ }^{1,5,6}$

It remains unclear which type of prosthesis is most suitable for small children. Mechanical valves typically have a low profile and good durability but carry the risk of thrombosis and embolization as well as bleeding associated with anticoagulation. ${ }^{2}$ Bioprosthetic valves obviate the need for long-term anticoagulation and might

- Scanning this $\mathrm{QR}$ code will take you to a supplemental video for the article. 


\section{Abbreviations and Acronyms \\ $\mathrm{BCH}=$ Boston Children's Hospital \\ LVAD $=$ left ventricular assist device \\ MVR $=$ mitral valve replacement}

offer more favorable hemodynamics but can calcify and fail rapidly, especially in young children. ${ }^{7,8}$ We investigated the mechanisms for bioprosthetic valve failure in very young patients who underwent MVR at Boston Children's Hospital (BCH) from 2010 to 2016, focusing on the macroscopic and microscopic findings in explanted valves.

\section{METHODS}

All patients who underwent MVR at BCH between January 2010 and April 2016 at $<5$ years of age were identified by search of the computerized Cardiac Surgical database. Patients who underwent MVR with a bioprosthetic valve (either porcine or bovine pericardial valves) were the subjects of this study. Patients receiving a Melody (Medtronic, Inc, Minneapolis, Minn) or a mechanical prosthesis were excluded. The institutional review board of BCH approved this study (IRB-P00022673).

Data were obtained by retrospective chart review of medical records, operative reports, clinic documentation, and echocardiogram reports and images. Valve failure was defined as a need for prosthetic MVR explantation. Pre-MVR baseline patient characteristics recorded included native anatomy, previous catheter-based and surgical interventions, age at implantation, and predominant mitral pathophysiology at the time of MVR. Valve parameters included type and size of valve, subsequent interventions, clinical history of dysfunction, and duration in situ. The time to most recent follow-up was recorded for all bioprosthetic valves still in situ at the time of this study.

Explanted valves were examined and photographed in the fresh state when possible. Valves were examined in situ at autopsy or after heart transplantation. Sections of leaflets from the free edge to the base were processed for histology. The sections were embedded in paraffin, cut into $5-\mu \mathrm{m}$ sections, mounted, and stained with hematoxylin and eosin and Masson trichrome stains.

\section{Statistical Analysis}

Patient and valve characteristics were summarized by the use of frequencies and percentages for categorical variables and medians with ranges for continuous variable. This was done for the cohort as a whole and separately for patients with valve failure and those with valve in situ. Time from MVR to valve failure (ie, valve explantation) was estimated with the Kaplan-Meier method. Differences in time to valve failure were compared for subgroups of patients via the log-rank test. Two MVRs in the same patient were assumed to be independent.

\section{RESULTS}

During the study period, 77 MVRs were performed in 55 children $<5$ years of age. Bioprosthetic valves were used in 31 MVRs $(40 \%$; 25 porcine, 6 bovine) (Table 1). A mechanical valve $(\mathrm{n}=16)$ or a Melody valve $(\mathrm{n}=30)$ was used for the remaining replacements. Median follow-up time was 10.5 months (range 1 week to 6 years). Six of the porcine valves were Carpentier-Edwards
TABLE 1. Bioprosthetic valve type

\begin{tabular}{lc}
\hline \multicolumn{1}{c}{ Prosthesis type } & Number \\
\hline Porcine valves & 25 \\
Carpentier-Edwards bioprosthetic valve & 4 \\
Carpentier-Edwards bioprosthetic valve conduit & 6 \\
St Jude Medical Epic Supra heart valve & 14 \\
Mosaic Medtronic tissue valve & 1 \\
Bovine pericardial valves & \\
Magna Ease aortic valve & 6 \\
\hline
\end{tabular}

bioprosthetic valves (Edwards Lifesciences, Irvine, Calif) within Dacron conduits. The Dacron conduit was removed or significantly trimmed down in each of these cases.

\section{Clinical History}

Characteristics of the 31 patients who received bioprosthetic mitral valves are summarized in Table 2 . The most common native congenital heart defects were congenital mitral stenosis with Shone complex $(n=8$, $26 \%)$, common atrioventricular canal defect $(\mathrm{n}=6$, $19 \%$ ), hypoplastic left heart syndrome with mitral stenosis $(\mathrm{n}=5,16 \%)$, and congenital mitral regurgitation $(\mathrm{n}=4$, $13 \%)$. All patients had at least 1 previous attempt at surgical mitral valve repair with a median of 2 surgeries (range 1-4 surgeries), and 12 patients (39\%) had previous MVR. Predominant pathophysiology at the time of MVR was mitral stenosis in 8 patients $(26 \%)$, mitral regurgitation in 17 patients (55\%), and mixed in 6 patients (19\%). Median age at MVR was 2.4 years (range 47 days- 4.9 years), and median size of bioprosthetic valve used was $19 \mathrm{~mm}$ (range $12-27 \mathrm{~mm}$ ).

Valve failure occurred in 10 patients $(32 \%)$. Freedom from valve failure was $80 \%$ (95\% confidence interval, 54-92) at 1 year (Figure 1). Shorter freedom from valve failure was found to be associated with younger age at implantation (Figure 2).

An echocardiogram within 1 month of implantation showed more than mild mitral stenosis in a single patient, mild mitral regurgitation in 4 patients, and a paravalvar leak in 3 patients. Early echocardiographic findings were not predictive of valve failure. After MVR, 24 patients $(77 \%)$ received therapeutic heparin (range of days 2 to $>30$ ), and within 1 month of implantation 22 patients were on aspirin, 4 patients were on warfarin (Coumadin), and 2 patients were on enoxaparin. There was no significant association between anticoagulation strategies with valve failure.

Of the 10 explanted valves, 8 were porcine (32\% of 25 implanted) and 2 bovine pericardial (33\% of 6 implanted). The median time to explantation was 1.4 years (range 56 days to 4.4 years). All 10 valve failures were due to prosthetic mitral stenosis, 7 of which were graded as severe on echocardiogram. Seven patients had at least 
TABLE 2. Baseline characteristics

\begin{tabular}{|c|c|c|c|c|}
\hline & Total $(\mathbf{n}=\mathbf{3 1})$ & No valve failure $(n=21)$ & Valve failure $(n=10)$ & $P$ value \\
\hline \multicolumn{5}{|l|}{ Age at implantation, $y$} \\
\hline$<1$ & $9(29 \%)$ & $4(19 \%)$ & $5(50 \%)$ & \multirow{3}{*}{.008} \\
\hline 1 to $<3$ & $13(42 \%)$ & $8(38 \%)$ & $5(50 \%)$ & \\
\hline 3 to $<5$ & $9(29 \%)$ & $9(43 \%)$ & $0(0 \%)$ & \\
\hline Median age at implantation, y, median (range) & $2.4(0.1-4.9)$ & $2.8(0.4-4.9)$ & $1.2(0.1-2.9)$ & $.01 *$ \\
\hline \multicolumn{5}{|l|}{ Cardiac diagnosis } \\
\hline CAVC & $6(19 \%)$ & $5(24 \%)$ & $1(10 \%)$ & \multirow{4}{*}{.14} \\
\hline CMS/Shone & $8(26 \%)$ & $5(24 \%)$ & $3(30 \%)$ & \\
\hline HLHS & $5(16 \%)$ & $3(14 \%)$ & $2(20 \%)$ & \\
\hline Other & $12(39 \%)$ & $8(38 \%)$ & $4(40 \%)$ & \\
\hline \multicolumn{5}{|l|}{ No. previous mitral valve repair } \\
\hline $1-2$ & $21(68 \%)$ & $12(57 \%)$ & $9(90 \%)$ & \multirow[t]{2}{*}{.07} \\
\hline $3-4$ & $10(32 \%)$ & $9(43 \%)$ & $1(10 \%)$ & \\
\hline \multicolumn{5}{|l|}{ Predominant physiology at MVR } \\
\hline Stenosis & $8(26 \%)$ & $8(38 \%)$ & $0(0 \%)$ & \multirow[t]{3}{*}{.19} \\
\hline Regurgitation & $17(55 \%)$ & $9(43 \%)$ & $8(80 \%)$ & \\
\hline Mixed & $6(19 \%)$ & $4(19 \%)$ & $2(20 \%)$ & \\
\hline \multicolumn{5}{|l|}{ Valve type } \\
\hline Porcine & $25(81 \%)$ & $17(81 \%)$ & $8(80 \%)$ & \multirow[t]{2}{*}{.43} \\
\hline Bovine pericardial & $6(19 \%)$ & $4(19 \%)$ & $2(20 \%)$ & \\
\hline \multicolumn{5}{|l|}{ Valve size, $\mathrm{mm}$} \\
\hline$<19$ & $9(29 \%)$ & $5(24 \%)$ & $4(40 \%)$ & \multirow{3}{*}{.24} \\
\hline 19 & $14(45 \%)$ & $9(43 \%)$ & $5(50 \%)$ & \\
\hline$>19$ & $8(26 \%)$ & $7(33 \%)$ & $1(10 \%)$ & \\
\hline
\end{tabular}

$P$ values calculated with the log-rank test to account for differences in follow-up time. $P$ values in bold indicate statistical significance. $C A V C$, Common atrioventricular canal defect; $C M S$, congenital mitral stenosis; $H L H S$, hypoplastic left heart syndrome; $M V R$, mitral valve replacement. *Wilcoxon rank sum test.

some degree of prosthetic regurgitation as well prior to explantation.

In addition to the explanted valves, 4 patients died with a bioprosthetic mitral valve in situ and 1 patient underwent orthotopic heart transplant after MVR. All 5 were porcine valves. Three of these 5 valves ( 2 at autopsy and 1 after transplantation) were available for examination. In no case was the cause of death directly related to the prosthetic valve. The 2 bioprosthetic valves examined at autopsy were functioning

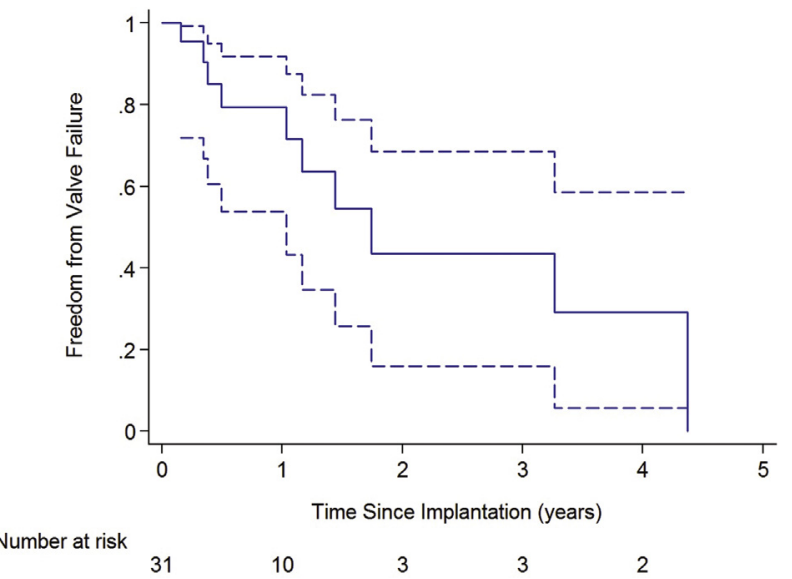

FIGURE 1. Kaplan-Meier curve showing overall freedom from valve failure after mitral valve replacement in patients $<5$ years of age. well when examined by echocardiography before death. The valve in the explanted heart developed moderate-to-severe regurgitation by the time of transplantation; this patient was supported by a left ventricular assist device (LVAD) implanted contemporaneously with the MVR.

\section{Gross and Histologic Pathology}

Porcine valves. There were 13 potential porcine valves for review: 8 that were explanted due to valve failure and 5 that

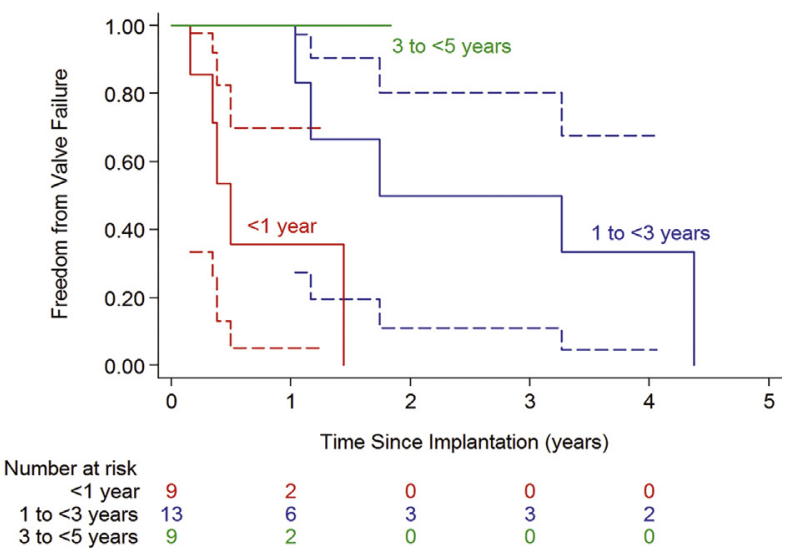

FIGURE 2. Freedom from valve failure by age at implantation. 


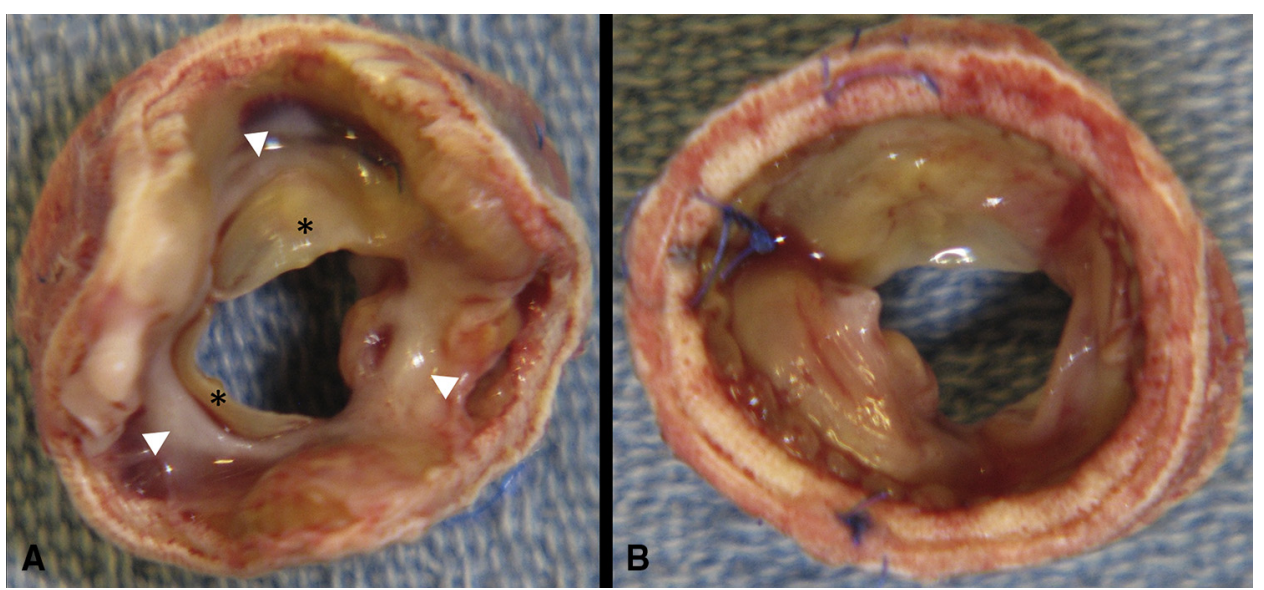

FIGURE 3. Ventricular (A) and atrial (B) surfaces of a porcine bioprosthesis explanted after 13 months in situ. The leaflets $(*)$ are thin but covered by thick pannus (white arrowheads) predominantly on the ventricular surface. Note that the 2 lower leaflets appear more affected than the upper one.

were from the patients that either died or underwent heart transplantation with the valve still in situ. Seven of the 8 explanted porcine valves were available for gross and histologic analysis. Six valves were intact, and the seventh contained a single intact leaflet with the other 2 leaflets partially missing (possibly due to operative artifact during extraction). On pathologic examination, valve dysfunction in all cases was due to pannus formation covering leaflets and/or intrinsic leaflet calcification. In 5 valves, pannus coated and stiffened at least one of the leaflets, causing it to be fixed and immobile, reducing the effective orifice size of the valve. In 3 valves, the pannus covered 2 of the leaflets. Four of the explanted porcine valves had pannus predominantly affecting the ventricular surface of the leaflet

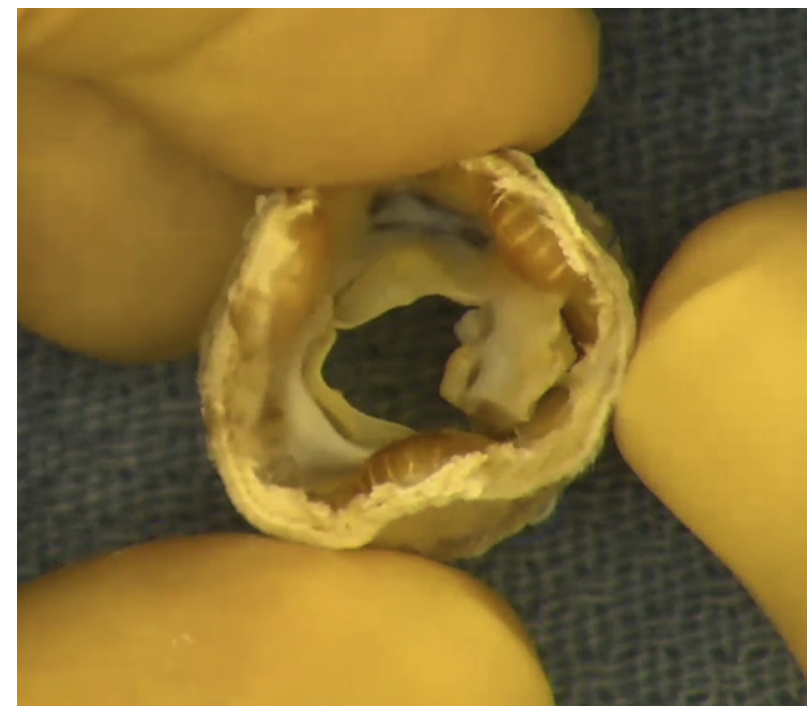

VIDEO 1. Demonstration of pannus formation on a porcine valve placed in the mitral position and explanted after 13 months. Video available at: http://www.jtcvsonline.org/article/S0022-5223(17)32132-3/fulltext. with minimal involvement of the atrial surface (Figure 3 and Video 1).

Histologic examination of these leaflets demonstrated that the pannus consisted of collagen with occasional elastic fibers and fibroblasts (Figure 4). In all but one of the valves, there were signs of thrombus formation with evidence of organization at the leading edge of the fibrous pannus proximal to the bare area of the valve leaflet (Figure 5).

Intrinsic leaflet calcification leading to at least one stiff and firm leaflet with limited mobility was found in 3 valves. The affected leaflets were densely and diffusely calcified, and there were associated calcific nodules extending to the leaflet surface (Figure 6). Sections from these valves showed dense intrinsic calcification in the center of the leaflet (Figure 7).

Of the 5 valves in situ at death or transplant, 3 (2 autopsy, 1 posttransplant) were available for review. The 2 valves examined at autopsy were in situ for 1 month and 1 week before death. On one of these valves, gross examination

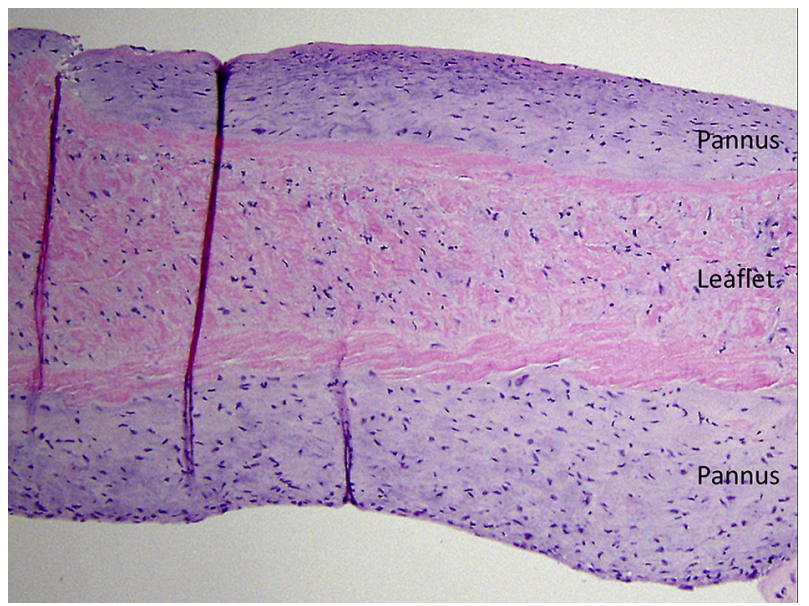

FIGURE 4. A hematoxylin and eosin-stained section at $\times 2$ magnification of a porcine valve leaflet covered with thick pannus. 


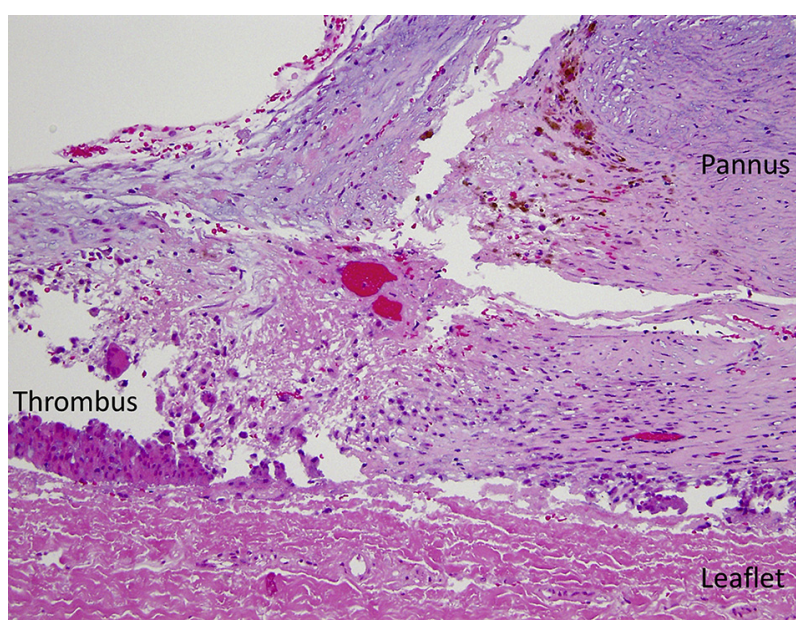

FIGURE 5. A section through a pericardial leaflet showing organizing thrombus between the leaflet and pannus.

showed no abnormality except a small paravalvar leak. Histologic examination of the valve in situ for 1 month revealed microscopic thrombus on the ventricular surface of the leaflets and along the free edge of the posteromedial leaflet without signs of infection, pannus formation, or calcification; the valve in situ for 1 week was histologically unremarkable. The third valve, in the explanted heart, was in situ for 5.5 months before heart transplantation, during which time a LVAD was also in place. On gross examination all 3 leaflets were diffusely encased in a layer of thick pannus on both the atrial and ventricular surfaces. The encased leaflets were firm and stuck in a partially opened position. Sections from this valve were consistent with organized thrombus and subsequent pannus formation.

Bovine pericardial valves. Both of the explanted bovine pericardial valves were available for gross and histologic examination. On gross examination, both valves had restricted movement of 2 leaflets secondary to pannus over growth on the ventricular surface. On one valve, the pannus growth diffusely covered 2 leaflets, fixing them in a partially opened position. On the atrial surface, there was pannus growth along the valve ring. On the other, pannus growth was concentrated at one strut and commissure with encroachment onto the two adjacent leaflet bellies. The 2 leaflets were fixed in a partially opened position. Histology of both valves was consistent with fibrous pannus, but evidence of preceding thrombus formation with organization was only found on the first valve.

\section{DISCUSSION}

Although repair remains the preferred approach for mitral valve disease, valve replacement is the only therapeutic option for cases in which repair is not feasible or does not yield lasting benefit. MVR in the first few years of life poses a challenge, mostly because of the small size of the mitral valve annulus, left ventricle, and left atrium. Early experience with bioprosthetic valves in the 1970s and early 1980s revealed limited valve longevity as the result of intrinsic calcification, a process that appeared to be accelerated in children. ${ }^{8-10}$ Advances in fixation and antimineralization processes ${ }^{11}$ have led to a resurgence of bioprosthetic valve use in children. In our small series, we found a substantial rate of early valve failure. Our study reaffirms that intrinsic calcification continues to limit the durability of some bioprosthetic valves in young patients and further reveals that pannus formation is another mechanism for early, rapid valve failure in this population.

Pannus can form as part of a host's reaction to surgical trauma, a foreign body, or as the end product of organized thrombus formation. Pannus formation is part of the healing process and can be beneficial as a nonthrombogenic layer covering prosthetic valves. However, in excess, it can lead to valve dysfunction with stenosis and/or regurgitation. ${ }^{12,13}$

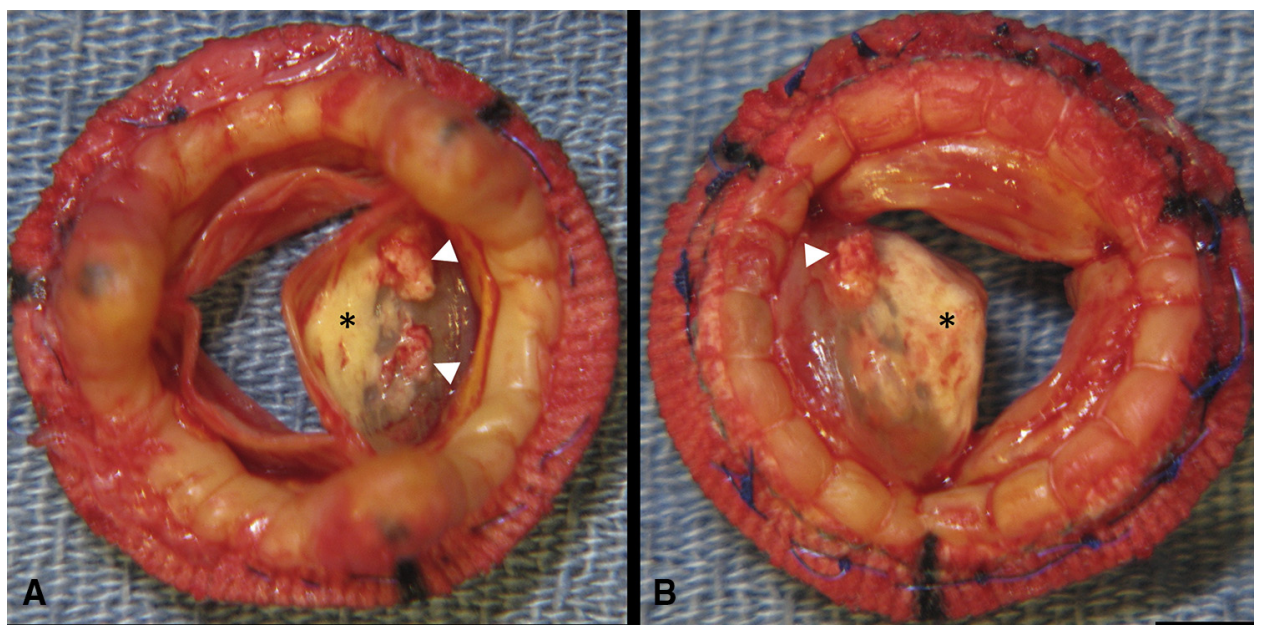

FIGURE 6. Ventricular (A) and atrial (B) surfaces of a porcine bioprosthesis explanted after 17 months in situ. There is dense intrinsic calcification of the belly of 1 leaflet $(*)$ with calcific nodules (white arrowheads) on the surface. 


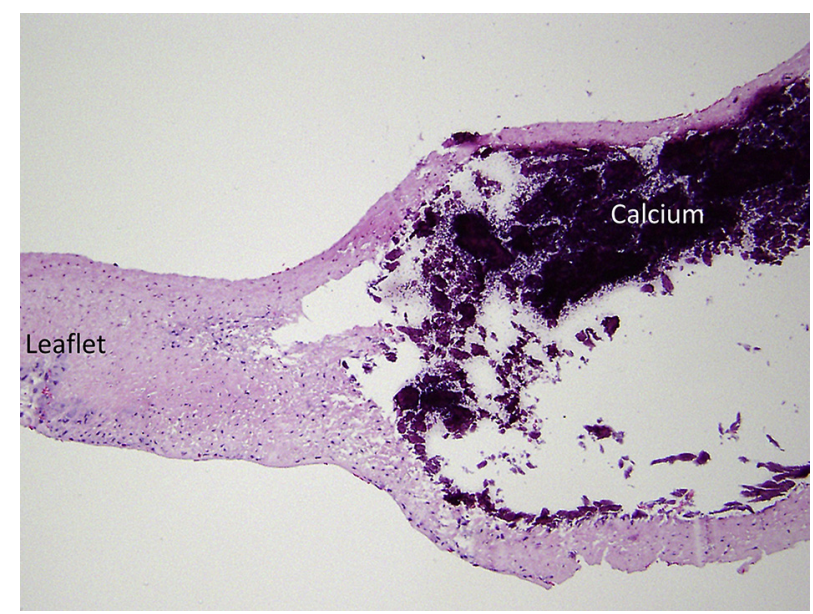

FIGURE 7. A hematoxylin and eosin-stained section at $10 \times$ magnification of a densely calcified porcine leaflet. The calcification is within the core of the leaflet (intrinsic calcification). The clear areas within the leaflet are due to loss of calcium during processing of the specimen.

Excessive pannus deposition leading to restricted leaflet mobility was noted in 8 of the 10 failed valves available for review. Pannus formation was predominantly located on the ventricular surface of leaflets in 4 of 5 explanted porcine valves and both bovine pericardial valves. We also observed that in most of these cases the pannus deposition affected 2 of the 3 leaflets. We hypothesize that the unaffected leaflet likely sat in the left ventricular outflow tract (anterior) and that high velocity flow across the ventricular aspect of the leaflet prevented thrombus deposition and subsequent exuberant pannus formation. In contrast, the 2 affected leaflets were adjacent to the posterior wall where blood flow is likely to be of lower velocity and without clear run-off, favoring thrombus deposition. Thrombus coating these leaflets becomes organized and then converted to fibrous pannus. Further evidence for this mechanism was seen in the valves at the time of autopsy or transplantation. The porcine valve examined at autopsy after 1 month in situ revealed small early thrombus deposition. The prosthesis implanted at the same time as an LVAD, was exposed to 5.5 months of continuous nonpulsatile flow, without a high velocity jet across the anterior leaflet because the ventricle was not ejecting. The continuous flow across the valve associated with the LVAD held it in a stable open position. In this case, all 3 leaflets became encased in a thick layer of pannus. In light of these findings of valve failure due to exuberant pannus formation in response to thrombus deposition, one could hypothesize that targeted anticoagulation could be beneficial. However, as this is a descriptive study, additional investigations would be needed to address the effects of anticoagulation in this population.

Our series suggests that the etiology of early excessive pannus growth, however, is likely multifactorial. We observed a second pattern with pannus extending from the valve ring onto the adjacent atrial surface of the valve strut with extension onto the adjacent leaflets, fusing them together at the commissure. In this location, a transition of fibrin thrombus to organized pannus was not observed between the fibrotic layer and valve leaflet. The pannus in this location appears to be due to the patient's inflammatory response to the foreign material with macrophages and foreign body giant cells, rather than to organization of thrombus. Bioprosthetic valves are treated with glutaraldehyde to reduce antigenicity, but risk of an immune reaction remains present and can contribute to calcification of the valve. This process is thought to play a role in the early calcification process seen in bioprosthetic valves implanted in children who are more immunocompentent. ${ }^{14-16}$

Although pannus deposition appeared to be the primary cause of valve failure in the majority of failed valves in this series, intrinsic leaflet calcification played a role in 3 explanted porcine valves. This finding highlights that calcification continues to be a limiting factor in the durability of bioprosthetic valves in very young patients.

This study is a small, single-institution experience with bioprosthetic valves in very young patients. An in situ description of the explanted valves is not available in most cases, so we do not know to what extent the gross appearance of the valves was altered by extraction. Because of the small number of specimens and heterogeneous patient population, our findings might not be generalizable.

\section{CONCLUSIONS}

Successful long-term use of bioprosthetic valves in the mitral position in young patients continues to be a challenge. In addition to the ongoing problem of intrinsic calcification, bioprosthetic valves pose the risk of early valve failure due to excessive pannus deposition. Early exuberant pannus growth appears to occur in response to thrombus deposition on the valves themselves as well as to the host's inflammatory reaction to foreign material. These mechanisms highlight the multifactorial etiology of valve failure and suggest future directions for further investigation into potential management strategies, such as longer anticoagulation, to prolong the durability of these valves in young patients.

\section{Conflict of Interest Statement}

Dr Paderais a pathology consultant for Medtronic. All other authors have nothing to disclose with regard to commercial support.

\section{References}

\footnotetext{
1. Caldarone CA, Raghuveer G, Hills CB, Atkins DL, Burns TL, Behrendt DM, et al. Long-term survival after mitral valve replacement in children aged <5 years: a multi-institutional study. Circulation. 2001;104:I143-7.
} 
2. Raghuveer G, Caldarone CA, Hills CB, Atkins DL, Belmont JM, Moller JH. Predictors of prosthesis survival, growth, and functional status following mechanical mitral valve replacement in children aged less than 5 years, a multi-institutional study. Circulation. 2003;108(suppl 1):II174-9.

3. Brown JW, Fiore AC, Ruzmetov M, Eltayeb O, Rodefeld MD, Turrentine MW. Evolution of mitral valve replacement in children: a 40-year experience. Ann Thorac Surg. 2012;93:626-33; discussion 633.

4. Eble BK, Fiser WP, Simpson P, Dugan J, Drummond-Webb JJ, Yetman AT Mitral valve replacement in children: predictors of long-term outcome. Ann Thorac Surg. 2003;76:853-9.

5. Alsoufi B. Aortic and mitral valve replacement in children: current options and outcomes. Expert Rev Cardiovasc Ther. 2011;9:805-9.

6. Alsoufi B, Manlhiot C, Al-Ahmadi M, McCrindle BW, Kalloghlian A, Siblini G, et al. Outcomes and associated risk factors for mitral valve replacement in children. Eur J Cardiothorac Surg. 2011;40:543-51.

7. Schoen FJ, Levy RJ. Calcification of tissue heart valve substitutes: progress toward understanding and prevention. Ann Thorac Surg. 2005;79: $1072-80$.

8. Antunes MJ. Bioprosthetic valve replacement in children-long-term follow-up of 135 isolated mitral valve implantations. Eur Hear J. 1984;5:913-8.

9. Sanders SP, Levy RJ, Freed MD, Norwood WI, Castaneda AR. Use of Hancock porcine xenografts in children and adolescents. Am J Cardiol. 1980; 46:429-38.
10. Silver MM, Pollock J, Silver MD, Williams WG, Trusler GA. Calcification in porcine xenograft valves in children. Am J Cardiol. 1980;45:685-9.

11. Chikwe J, Filsoufi F. Durability of tissue valves. Semin Thorac Cardiovasc Surg. 2011;23:18-23.

12. Donegani E, Barberis RV, Marco R. Morphologic findings in explanted pericardial bioprostheses in young patients. Int J Cardiovasc Res. 2013;2:2-6.

13. Cremer PC, Rodriguez LL, Griffin BP, Tan CD, Rodriguez ER, Johnston DR, et al. Early bioprosthetic valve failure: mechanistic insights via correlation between echocardiographic and operative findings. I Am Soc Echocardiogr. 2015;28:1131-48.

14. Côté N, Pibarot P, Clavel M-A. Incidence, risk factors, clinical impact, and management of bioprosthesis structural valve degeneration. Curr Opin Cardiol. 2017:32:123-9.

15. Park CS, Park SS, Choi SY, Yoon SH, Kim WH, Kim YJ. Anti alpha-gal immune response following porcine bioprosthesis implantation in children. J Heart Valve Dis. 2010;19:124-30.

16. Manji RA, Zhu LF, Nijjar NK, Rayner DC, Korbutt GS, Churchill TA, et al. Glutaraldehyde-fixed bioprosthetic heart valve conduits calcify and fail from xenograft rejection. Circulation. 2006;114:318-27.

Key Words: mitral valve replacement, bioprosthetic valve, pediatrics 